\title{
Identification of Wave Energy Device Models From Numerical Wave Tank Data-Part 2: Data-Based Model Determination
}

\author{
Simone Giorgi, Josh Davidson, and John V. Ringwood, Senior Member, IEEE
}

\begin{abstract}
In this paper and its companion [1], the identification of mathematical models describing the behaviour of wave energy devices (WECs) in the ocean is investigated through the use of numerical wave tank experiments. When the wave amplitude and the WEC displacement are not negligible with respect to the WEC dimensions, nonlinear hydrodynamic effects may appear, and the accuracy of linear hydrodynamic models is reduced, leading to the necessity of introducing some nonlinearities in the model structure. This paper proposes, for WEC modelling, the use of discrete-time nonlinear autoregressive with exogenous input (NARX) models, as an alternative to continuous-time models. Techniques of model identification are also explained and applied to a case study.
\end{abstract}

Index Terms-System identification, numerical wave tank, wave energy, discrete-time modelling, NARX model, ARX model, artificial neural network, Kolmogorov-Gabor polynomial model.

\section{INTRODUCTION}

M ATHEMATICAL modelling of wave energy devices has many uses, including the simulation of device motion, power production assessment and as a basis for model-based control design. In all these cases, it is important to describe how the body moves in the water, interacting with the waves propagating on the fluid surface. From a modelling point of view, this corresponds to the description of a mathematical relationship between the free surface elevation (FSE) of the water, $\eta$, (a model input) and the body displacement from an equilibrium position, $y$, (the model output). In the present paper, the family of all models having $\eta$ as input and $y$ as output is indicated with the symbolism $\eta \rightarrow y$. In a previous study, [2] employed a state-space structure for a model belonging to $\eta \rightarrow y$.

A large number of models employed in the simulation and analysis of WECs are based on the Cummins' equation [3]. Cummins' equation is based on Newton's second law, describing the motion of the device of mass $M$, floating in water, subject to fluid, gravity and other external forces (such as a mooring force, $f_{M}$, and a PTO force, $f_{P T O}$ ). The fluid force is derived under the simplifying hypothesis of linear potential

Manuscript received July 31, 2015; revised November 26, 2015; accepted January 01, 2016. This work was supported in part by the Enterprise Ireland under Grant EI/CF/2011/1320, in part by the Irish Government and the European Union under Ireland's EU Structural and Investment Funds Programme 2014-2020, and in part by the Science Foundation Ireland under Grant 13/IA/1886. Paper no. TSTE-00635-2015.

The authors are with the Centre for Ocean Energy Research, Maynooth University, Maynooth, Ireland (e-mail: john.ringwood@eeng.nuim.ie).

Color versions of one or more of the figures in this paper are available online at http://ieeexplore.iee.org.

Digital Object Identifier 10.1109/TSTE.2016.2515500 theory, which allows the total fluid force (applied from the fluid to the floating body) to be written as the summation of the excitation force, $f_{E}$, the radiation force, $f_{R A D}$, and the buoyancy force, $f_{B U}$, and to express the radiation force as a convolution integral. The Cummins' equation can be written as:

$$
\left(M+m_{\infty}\right) \ddot{y}(t)+\int_{-\infty}^{t} h_{R A}(t-\tau) \dot{y}(\tau) d \tau+K y(t)=f_{I N}(t)
$$

where $f_{I N}=f_{M}+f_{E}+f_{P T O}$ represents the model input, $y$ the model output (see Fig. 1), $f_{E}$ the excitation force from the fluid, $h_{R A}$ the radiation impedance impulse response function minus $m_{\infty}$ (the infinite frequency added mass), and $K$ the restoring coefficient.

In this paper, the family of all models having $f_{I N}$ as input and $y$ as output is indicated with the symbolism $f_{I N} \rightarrow y$. Different studies have been already conducted for the $f_{I N} \rightarrow$ $y$ model family, with [2] utilising a linear state-space, while [4] and [5] provide both frequency- and time-domain descriptions. These previous studies utilise models characterized by a linear relationship between input and output. Linear models have very desirable properties, such as superposition or a frequency domain description, but they are based on the hypothesis of small waves, ideal fluid (inviscid and incompressible) and small body displacement, which are not usually satisfied in reality. Consequently, when the wave amplitude and the body displacement increase (becoming of the same order of magnitude of the dimensions of the body), some nonlinear effects may appear. Examples of nonlinear hydrodynamic models have been developed by introducing an additional viscous nonlinear term [6], nonlinear Froude-Krylov force modelling [7] [8] [9] or nonlinear restoring force representation [10].

An alternative modelling approach, utilised in this paper for both $\eta \rightarrow y$ and $f_{I N} \rightarrow y$ model families, is that of system identification, where models are determined from input/output data, measured from the system under study [11]. Such methods are particularly useful where the system to be modelled is very complex and/or does not easily lend itself to first principles modelling. However, one major difficulty in system identification is ensuring that the input/output data, used to determine the model, is sufficiently representative of the system dynamics and, in particular, must cover the range of frequencies and amplitudes likely to be encountered during system operation. In the WEC case, such a range of excitation signals are not likely to be available in the open ocean (at least not in a reasonably short time frame) and, even more importantly, there is no 


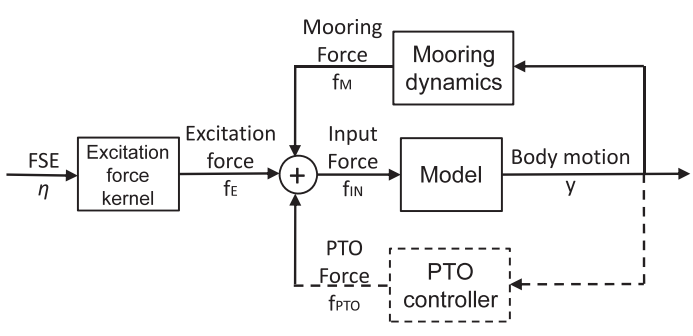

Fig. 1. Block diagram for the $f_{I N} \rightarrow y$ family model.

external control of the excitation. An alternative possibility is to employ tank tests. However, in addition to the significant cost and the need for a physical prototype, there may be limitations on the range of excitation signals available and tank wall reflections may further limit the range and duration of viable tests. A different option for generating suitable input/output data is to use a numerical wave tank (NWT), as explained in a companion paper [1].

In the current paper, for simplicity, the general 6 degree of freedom (DoF) problem has been reduced to a heave single DoF. However, the illustrated methods and procedures can be extended to the full $6 \mathrm{DoF}$, obtaining identified parametric models, which are computationally efficient in subsequent simulation and control design use. Indeed, the parametric model structures are able to capture the essential nonlinearities, remaining, simultaneously, with a sufficient simplicity, that allows the models to be run in real time. The price of the demanding NWT computation time is paid only once, for the simulation of the identification experiments.

The paper is laid out as follows; in Section II, the structure of the proposed linear and nonlinear models is outlined. Section III then briefly explains the NWT experiments used to produce the system identification data (with a fully detailed description available in [1]). Section IV describes the process of identifying the model parameters from the NWT generated data. Some illustrative examples demonstrating these methods are then given in Section V, and the results of the different models compared. Conclusions are drawn in Section VI.

\section{Model Parametric Structures}

The choice of the parametric structure of the model is very important, in the identification of the relationship between the model input and output. The model structure may be inspired from physical system knowledge and considerations (whitebox modelling) or completely based on the recorded data itself (black-box modelling). There are also many possibilities within these two extremes which are denoted by shades of grey e.g. off white, slate grey, smoke grey, etc [12]. In general, whether the model structure is inspired by physical phenomena or purely derived from the data, it is necessary to determine a parsimonious structure for the model [13], which will work well with the training data, but also generalise well to other data. A compatible requirement is that it is not desirable to add complexity to the model for little gain. The identified parametric model should also be able to capture the essential

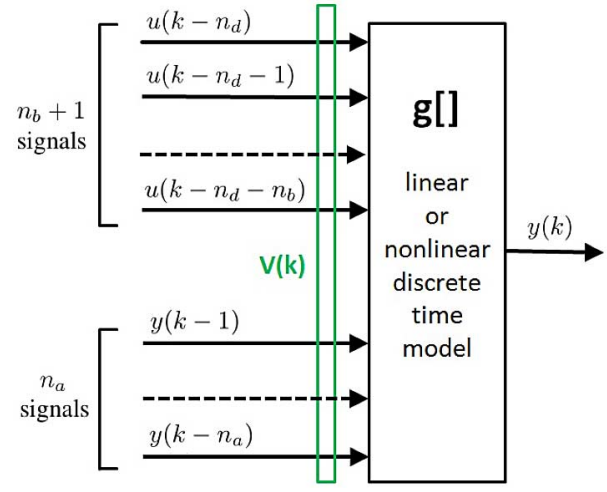

Fig. 2. Block diagram for a general NARX discete-time model.

nonlinearities, but retain sufficient simplicity that allows the model to be run in real time.

In recent years, a number of continuous-time models have been developed [14] [15], but, in this Section, a family of discrete-time models are proposed as a new alternative for WEC modelling.

\section{A. Discrete-Time Models}

Considering the discrete time nature of sampled data from experiments, the majority of system identification techniques are based on discrete time models [11]. Discrete-time modelling uses signals only specified at the discrete time instants $t=k T_{s}$, where $T_{s}$ is the sampling period and $k$ is an integer. For example, the values of $\eta, f_{I N}$ and $y$ at the time instant $k T_{s}$ are represented with the symbols $\eta(k), f_{I N}(k)$ and $y(k)$, respectively. In the NWT, little 'noise', barring some small numerical error, is present; therefore, there is no reason to provide a coloured noise model structure.

The relationship between $\eta$ and $y$ is non-causal [16], therefore, noncausality has to be introduced into the structure of the identified model. In this paper, nonlinear autoregressive with exogenous input (NARX) models [17] are utilized. In NARX models, the present value of the output $y(k)$ depends on the past values of the output $y(k-1), \ldots, y\left(k-n_{a}\right)$ and the input values $u\left(k-n_{d}\right), u\left(k-n_{d}-1\right), \ldots, u\left(k-n_{d}-n_{b}\right)$. If the system is causal, the output does not depend on future values of the input and $n_{d} \geq 0$, otherwise, if the system is noncausal, $n_{d}<0$ and the present value of the output is influenced by future input values. The NARX model is summarized with the following equation:

$$
\begin{aligned}
y(k)= & g\left[y(k-1), \ldots, y\left(k-n_{a}\right),\right. \\
& \left.u\left(k-n_{d}\right), u\left(k-n_{d}-1\right), \ldots, u\left(k-n_{d}-n_{b}\right)\right]
\end{aligned}
$$

which can be represented with the block diagram of Fig. 2 . $n_{a}$ and $n_{b}$ represent the dynamical order of the model, and by increasing them, the model becomes more flexible and able to show more complex dynamical behaviour but, at the same time, unnecessarily high orders can make the model less able to generalise on new data (overfitting). $n_{d}$ is the input delay time and it represents the number of samples before the output reacts to the input (for $n_{d} \geq 0$ ) or the number of future input steps that influence the present value of the output (for $n_{d}<0$ ). 
In equation (1), the generalised input $u$ is replaced with $\eta$, for a model belonging to the model class $\eta \rightarrow y$, and with $f_{I N}$, for a model belonging to $f_{I N} \rightarrow y$. The output $y$, in the present paper, always represents the body displacement.

In the current paper, three model structures are investigated: A linear autoregressive with exogenous input (ARX) model, the Kolmogorov-Gabor polynomial (KGP), which is a natural polynomial extension of the ARX model and an artificial neural network (ANN) model, which provides a structure-free form with further nonlinear modelling freedom (though with a nonconvex optimisation problem). Linear ARX models provide a reference linear model, with which nonlinear KGP and ANN models can be compared.

\section{B. Linear ARX Model}

The first parametric model examined is the autoregressive with exogenous input model. It is a well-known black box model with a linear input/output relationship [11] and it is linear in the parameters $a_{i}$ and $b_{i}$. For the ARX model, equation (1) becomes:

$$
y(k)=\sum_{i=1}^{n_{a}} a_{i} y(k-i)+\sum_{i=0}^{n_{b}} b_{i} u\left(k-n_{d}-i\right)
$$

and the number of parameters to be estimated is $N_{p a r}=n_{a}+$ $n_{b}+1$.

\section{Nonlinear Kolmogorov-Gabor Polynomial Model}

The second model considered in this paper utilises a polynomial nonlinearity, which results in a Kolmogorov-Gabor polynomial model [17]. In this case, equation (1) becomes:

$$
\begin{aligned}
y(k)= & \sum_{i=1}^{n_{a}} a_{i 1} y(k-i)+\sum_{i=0}^{n_{b}} b_{i 1} u\left(k-n_{d}-i\right) \\
& +\ldots \\
& +\sum_{i=1}^{n_{a}} a_{i p} y^{p}(k-i)+\sum_{i=0}^{n_{b}} b_{i p} u^{p}\left(k-n_{d}-i\right) \\
& +\sum_{i=1}^{n_{a}} \sum_{j=0}^{n_{b}} c_{i j} y(k-i) u\left(k-n_{d}-j\right) \\
& +\ldots
\end{aligned}
$$

where $p$ is the maximum polynomial order for the terms involving $u$ and $y$ alone, as well as the cross-product terms.

The KGP model is a black box model having a nonlinear input/output relationship, but is linear in the parameters $a_{i j}, b_{i j}$ and $c_{i j}$. However, the presence of the cross-product terms (between $u$ and $y$ ) in the KGP model structure can lead to potential, and unpredictable, stability problems. In essence, cross-product terms introduce input-dependent terms into the (notionally linearised) model Jacobian, which make the stability properties dependent on the input used. In fact, some experiences with cross-product terms showed the model response on validation data to be clearly unstable, even though the model response on training data was stable, in spite of the fact that the spectral and distribution characteristics of training and validation data were similar. For this reason, the crossproduct terms in the model of equation (2) have been removed, obtaining the simpler model:

$$
y(k)=\sum_{j=1}^{p}\left[\sum_{i=1}^{n_{a}} a_{i j} y^{j}(k-i)+\sum_{i=0}^{n_{b}} b_{i j} u^{j}\left(k-n_{d}-i\right)\right]
$$

In the presence of no cross-product terms, the number of parameters to be estimated for the KGP model is $N_{\text {par }}=$ $p\left(n_{a}+n_{b}+1\right)$.

\section{Artificial Neural Network Model (Nonlinear)}

The final parametric model utilised is a multi-layer perceptron (MLP) artificial neural network, [17], which is a black box model having a nonlinear input/output relationship and is nonlinear in the parameters. While one hidden layer of nonlinear neurons can give an arbitrary approximation capability, two hidden layers usually requires a lower total neuron count, for a given level of approximation accuracy. The overall model complexity is determined by $n_{1}$ and $n_{2}$, the number of neurons in the hidden layers 1 and 2, respectively, in addition to $n_{a}$ and $n_{b}$. The feedforward MLP is defined by $y(k)=g[V(k)]$ as in Fig 2, where:

$$
\begin{aligned}
V(k)= & {\left[v_{1}(k), v_{2}(k), \ldots, v_{n_{v}}(k)\right] } \\
= & {\left[y(k-1), \ldots, y\left(k-n_{a}\right),\right.} \\
& \left.u\left(k-n_{d}\right), u\left(k-n_{d}-1\right), \ldots, u\left(k-n_{d}-n_{b}\right)\right]
\end{aligned}
$$

$n_{v}=n_{a}+n_{b}+1$ is the number of variables. In this way, the input/output relationship of the ANN model can be written [17] as:

$$
y(k)=\sum_{i=0}^{n_{2}} w_{i}^{(\text {out })} \Psi_{i}\left(\sum_{j=0}^{n_{1}} w_{i j}^{(2)} \Psi_{j}\left(\sum_{l=0}^{n_{v}} w_{j l}^{(1)} v_{l}(k)\right)\right)
$$

where $v_{0}(k)=1, w_{j l}^{(1)}, w_{i j}^{(2)}$, and $w_{i}^{(o u t)}$ are the unknown parameters of hidden layer 1, hidden layer 2 and the output layer respectively, and $\Psi_{i}$ is a tan-sigmoid function defined as:

$$
\Psi_{i}(z)= \begin{cases}1 & \text { if } i=0, \\ 2 /\left(1+e^{-2 z}\right)-1 & \text { if } i \neq 0 .\end{cases}
$$

For the ANN model, the number of parameters to be estimated is $N_{\text {par }}=\left(n_{a}+n_{b}+2\right) n_{1}+\left(n_{1}+1\right) n_{2}+n_{2}+1$.

It is important to underline that, the nonlinearity in the parameters is a significant disadvantage of the ANN model compared to the ARX and KGP models, as it makes the determination of the optimal parameters considerably more difficult.

\section{NumericAl WAVE TANK}

A NWT is the generic name for numerical simulators of nonlinear free surface waves, hydrodynamic forces and floating body motions. NWTs can be implemented using boundaryelement methods or computational fluid dynamics (CFD). 


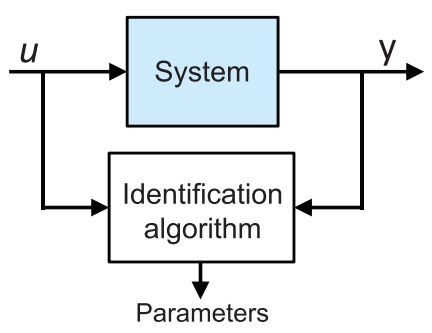

Fig. 3. System identification principle.

CFD-based NWTs offer fully nonlinear hydrodynamic calculations and solve the Navier-Stokes equations, which govern the transfer of mass, momentum and heat, by discretizing the domains of space and time to form a system of linear algebraic equations, which are computer implementable. The NWT utilised in the present paper is implemented using CFD and is detailed in [1].

\section{A. NWT Experiments}

1) Wave Excitation Experiments $(\eta \rightarrow y)$ : In this case, the excitation on the body is provided exclusively by the waves generated in the NWT, no PTO force is applied $\left(f_{P T O}=0\right)$, no mooring is present $\left(f_{M}=0\right)$ and the body displacement is measured. Two data vectors are produced by this experiment for model identification, one containing the FSE, $\{\eta(k)\}$, and the other the body displacement, $\{y(k)\}$.

2) Input Force Experiments $\left(f_{I N} \rightarrow y\right)$ : In this case, the only excitation on the body is the force applied by the PTO $\left(f_{I N}=f_{P T O}\right)$, no waves are generated in the NWT $\left(f_{E}=0\right)$, no mooring is present $\left(f_{M}=0\right)$ and the body displacement is measured. Two data vectors are also produced by this experiment for model identification, one containing the PTO force, $\left\{f_{I N}(k)\right\}$, and the other the body displacement, $\{y(k)\}$.

For the details regarding the NWT experiments [1].

\section{MOdEL IDENTIFICATION}

\section{A. System Identification}

The fundamental idea of system identification is represented in Fig. 3. The identification procedure is based on the following sequence of steps:

1) A parametric structure is chosen for the model,

2) A suitable input signal, $u$, is synthesised and input to the system,

3) The input signal, $u$, and resulting output signal, $y$, are recorded,

4) An identification algorithm is used to determine the optimal parameter vector, $\theta$, which minimises some error metric between the actual measured output $y$ and that produced by the identified parametric model, $\hat{y}$.

\section{B. Linear Regression and Least Squares}

Consider a model with an output, $\hat{z}$, which is a linear combination of $q$ variables, $x_{j}$ :

$$
\hat{z}=\theta_{1} x_{1}+\cdots+\theta_{q} x_{q}
$$

where the coefficients, $\theta_{j}$, are the unknown parameters. It will be assumed that, $N$ data samples have been measured so that the difference at each sample between the measured data and the model prediction is:

$$
\varepsilon=\mathbf{z}-\hat{\mathbf{z}}=\mathbf{z}-\mathbf{X} \boldsymbol{\theta}
$$

where:

$$
\begin{aligned}
& \varepsilon=\left[\begin{array}{llll}
\varepsilon(1) & \varepsilon(2) \ldots \varepsilon(N)
\end{array}\right]^{T}, \\
& \mathbf{z}=\left[\begin{array}{llll}
z(1) & z(2) & \ldots & z(N)
\end{array}\right]^{T}, \\
& \hat{\mathbf{z}}=\left[\begin{array}{llll}
\hat{z}(1) & \hat{z}(2) & \ldots & \hat{z}(N)
\end{array}\right]^{T}, \\
& \mathbf{X}=\left[\begin{array}{cccc}
x_{1}(1) & x_{2}(1) & \ldots & x_{q}(1) \\
x_{1}(2) & x_{2}(2) & \ldots & x_{q}(2) \\
\vdots & \vdots & \ddots & \vdots \\
x_{1}(N) & x_{2}(N) & \ldots & x_{q}(N)
\end{array}\right] \\
& \boldsymbol{\theta}=\left[\begin{array}{llll}
\theta_{1} & \theta_{2} & \ldots & \theta_{q}
\end{array}\right]^{T} .
\end{aligned}
$$

$\mathbf{X}$ and $\mathbf{z}$ are called the data matrix and the observation vector respectively. It's well known that the estimated parameters, which minimize the least squares (LS) error, are [17]:

$$
\hat{\boldsymbol{\theta}}=\underset{\theta}{\operatorname{argmin}}\left(\varepsilon^{T} \boldsymbol{\varepsilon}\right)=\left(\mathbf{X}^{\mathrm{T}} \mathbf{X}\right)^{-1} \mathbf{X}^{\mathrm{T}} \mathbf{z} .
$$

The least squares problem is not calculated directly from equation (6), because the use of $\mathbf{X}^{\mathrm{T}} \mathbf{X}$ increases the possibility of obtaining an ill-conditioned problem (the condition number of $\mathbf{X}^{\mathrm{T}} \mathbf{X}$ is approximately the square of the condition number of the data matrix $\mathbf{X}$ ). Instead, a QR factorization method is implemented, which computes the least squares solution directly from $\mathbf{X}$ without forming $\mathbf{X}^{\mathrm{T}} \mathbf{X}$ [18] [19].

\section{Time Delay and Dynamical Order Estimation $\left(n_{d}, n_{a}, n_{b}\right)$}

An important part of the model structure selection is the choice of the delay $n_{d}$ and the dynamical orders $n_{a}$ and $n_{b}$. As equation (1) suggests, $y(k)$ is a function of $n_{a}$ output and $n_{b}+1$ input values, taken at different time instants. Once $n_{a}$, $n_{b}$ and $n_{d}$ are selected, it is possible to obtain the different model structures (for this paper ARX, KGP and ANN) by changing the function $g[]$. The time delay and dynamical orders are estimated by implementing a systematic trial and error process on several ARX models, with varying $n_{a}, n_{b}$ and $n_{d}$, and selecting the values which give the best model performance, as measured by a loss function (a measure of the modelling error). For each ARX model estimation, independent training and validation data sets are utilised. At the end, the simplest ARX model able to repeat the validation data with a sufficient accuracy is selected (parsimonious model) [20].

In general, there are not straightforward methodologies to calculate the dynamical order and delay time of a nonlinear models (while relatively straightforward procedures exist for linear models). Therefore, the estimated $n_{a}, n_{b}$ and $n_{d}$ for the ARX model, are also utilised with the KGP and ANN models. Maintaining the same dynamical order and delay time for the linear and nonlinear models provides the possibility to isolate the comparative linear/nonlinear structure performances. 


\section{ARX Model Identification}

Performing the NWT wave excitation experiment (see Section III-A), the signals $\{\eta(k)\}$ and $\{y(k)\}$ for $k=1 \ldots N$, are generated, and are utilised as input and output for the identification of the ARX model belonging to $\eta \rightarrow y$. Defining $\tau=\max \left\{n_{a},\left(n_{b}+n_{d}\right)\right\}$, the first possible predicted model output is for $k=\tau+1$ (the first $\tau$ values of the data are utilised as initial conditions). Defining:

$$
\widetilde{N}= \begin{cases}N & \text { if } n_{d} \geq 0 \\ N+n_{d} & \text { if } n_{d}<0\end{cases}
$$

the last possible predicted model output is for $k=\tilde{N}$. Therefore, for an ARX model belonging to $\eta \rightarrow y$, equations (3), (4) and (5) become:

$$
\begin{aligned}
& \mathbf{z}=\left[\begin{array}{llll}
y(\tau+1) & y(\tau+2) \ldots y(\tilde{N})
\end{array}\right]^{T}, \\
& \mathrm{X}= \\
& {\left[\begin{array}{cccccc}
y(\tau) & \ldots y\left(\tau+1-n_{a}\right) & \eta\left(\tau+1-n_{d}\right) & \ldots \eta\left(\tau+1-n_{d}-n_{b}\right) \\
y(\tau+1) & \ldots y\left(\tau+2-n_{a}\right) & \eta\left(\tau+2-n_{d}\right) & \ldots & \eta\left(\tau+2-n_{d}-n_{b}\right) \\
\vdots & \ddots & \vdots & \vdots & \ddots & \vdots \\
y(\widetilde{N}-1) & \ldots & y\left(\widetilde{N}-n_{a}\right) & \eta(N) & \ldots & \eta\left(N-n_{b}\right)
\end{array}\right]} \\
& \hat{\theta}_{\mathrm{arx}}=\left[\begin{array}{llllllll}
a_{1} & a_{2} & \ldots & a_{n_{a}} & b_{0} & b_{1} & \ldots & b_{n_{b}}
\end{array}\right]^{T} .
\end{aligned}
$$

As explained in Section IV-B, the estimated parameters are determined by employing a QR factorization [18], [19].

Similarly, utilising the NWT input force experiment (see Section III-A), the signals $\left\{f_{I N}(k)\right\}$ and $\{y(k)\}$ for $k=1 \ldots N$, are generated, and are utilised for the identification of the ARX model belonging to $f_{I N} \rightarrow y$.

\section{E. Kolmogorov-Gabor Polynomial Model Identification}

Utilising the NWT wave excitation experiment signals for the identification of the KGP model belonging to $\eta \rightarrow y$, equations (3) and (5) become:

$$
\begin{gathered}
\mathbf{z}=[y(\tau+1) y(\tau+2) \ldots y(\tilde{N})]^{T}, \\
\hat{\theta}_{\mathbf{K G P}}=\left[a_{11}, \ldots, a_{n_{a} 1}, b_{01}, \ldots, b_{n_{b} 1}, \ldots, a_{1 p}, \ldots, a_{n_{a} p},\right. \\
\\
\left.b_{0 p}, \ldots, b_{n_{b} p}\right]^{T}
\end{gathered}
$$

For the KGP model, each of the $(\widetilde{N}-\tau)$ rows of the data matrix (4) has the form: $\left[y(k-1), \ldots, y\left(k-n_{a}\right), \eta\left(k-n_{d}\right), \ldots, \eta(k-\right.$ $\left.n_{d}-n_{b}\right), \ldots, y^{p}(k-1), \ldots, y^{p}\left(k-n_{a}\right), \eta^{p}\left(k-n_{d}\right), \ldots, \eta^{p}(k-$ $\left.\left.n_{d}-n_{b}\right)\right]$, where $k=(\tau+1), \ldots, \widetilde{N}$. The estimated parameters are determined by utilising a QR factorization algorithm (see Section IV-B). In similar way, the models belonging to $f_{I N} \rightarrow y$ are identified from the NWT input force experiments.

\section{F. Artificial Neural Network Model Identification}

Unlike the ARX and KGP models, the ANN model is nonlinear in the parameters; therefore, it is not possible to use the procedure, developed for linear regression in Section IV-B,

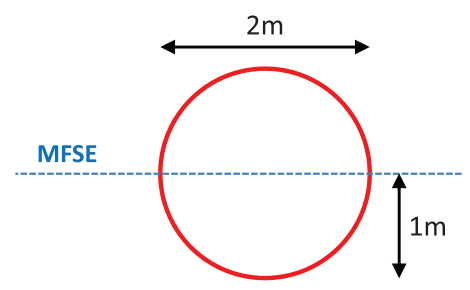

Fig. 4. 2D test device geometries (circle with 50\% draft). The dotted line represents the mean free surface elevation (MFSE).

for parameter identification. The nonlinear optimization problem, in this case, is solved utilising a scaled conjugate gradient algorithm [21]. Starting from a random initial value of the parameters, at each iteration (also denoted epoch), all of the training set is utilised to calculate the updated value of the parameters (batch training method). The solution is strongly dependent on the initial value due to the nonconvexity of the optimisation problem; therefore, 100 different initial conditions are employed to help determine an appropriate local minimum. The ability of the identified ANN model to generalise on new data is degraded in the case of training data overfitting; consequently, an early stopping technique is utilised, halting the training as soon as the error on the validation data begins to rise [22]. The numbers of neurons, $n_{1}$ and $n_{2}$, are determined by growing the network incrementally, until a good balance is achieved between approximation of the training data and generalisation capability.

\section{CAse Study Description}

The case study considers a two dimensional (2D) NWT (see [1] for details); therefore, the equivalent geometry of the test device is an infinitely long horizontal bar, having a vertical cross-section of a $2 \mathrm{~m}$ diameter circle, with a $1 \mathrm{~m}$ draft, as shown in Fig. 4. The variable cross-sectional area of the device geometry makes its hydrodynamic description challenging. The implemented NWT is a $50 \mathrm{~m}$ deep tank with walls $100 \mathrm{~m}$ from the device and with wave creation/absorption implemented via the waves2FOAM package utilising two $90 \mathrm{~m}$ long relaxation zones situated $5 \mathrm{~m}$ either side of the device [1].

As explained in the companion paper [1], in the experiment design, care must be taken to use excitation signals which provide an adequate distribution across the amplitude/frequency space. For the identification of models belonging to $\eta \rightarrow y$ and representative of a specific location, an excitation which covers the range of possibilities at the specific location should be chosen. However, the focus of the paper is to show the new methodology, rather than provide a complete description of any particular location/device combination and, for simplicity, two different NWT wave excitation experiments $W 1$ (for training) and $W 2$ (for validation) have been created, where the input wave signals are two different realizations of a commonly occurring sea state at the reference European Marine Energy Centre (EMEC) test site (significant wave height $H_{s}=0.6 \mathrm{~m}$ and peak period $T_{p}=8 \mathrm{~s}$ ) [1]. The input wave signals are multisine signals, consisting of 100 equally spaced frequencies in the range $0.005-0.995 \mathrm{~Hz}$, with randomly assigned phases 


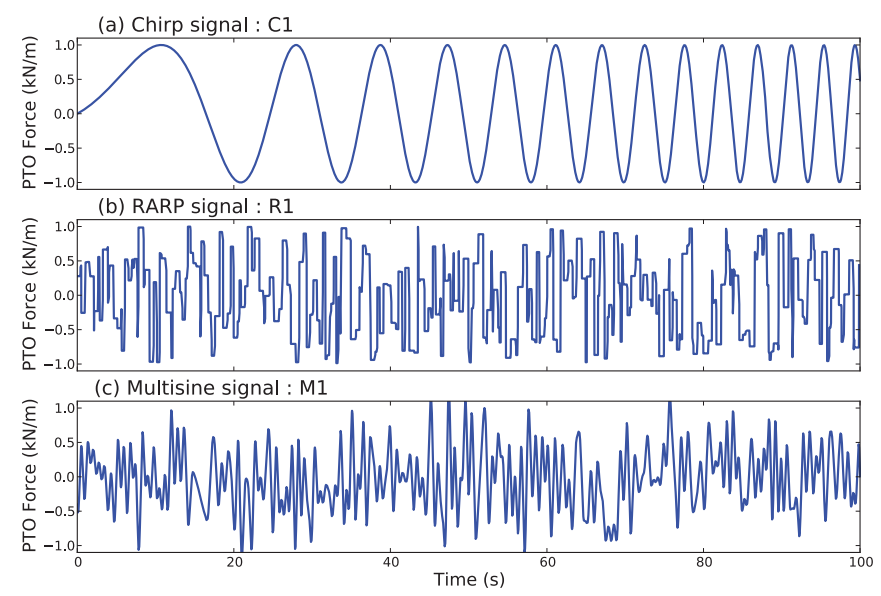

Fig. 5. 100 second portion of the 600 second input force signals used for model identification.

and amplitudes determined from a JONSWAP spectrum with peakiness $\gamma=2[1]$.

The identification and validation of the models belonging to $f_{I N} \rightarrow y$, are carried out using different NWT input force experiments:

- Two different Chirp signals $C 1$ (for training) and $C 2$ (for validation) with a maximum force amplitude of $1 \mathrm{kN} / \mathrm{m}$. $C 1$ sweeps the frequencies from $0 \mathrm{~Hz}$ up to $1.5 \mathrm{~Hz}$ and $C 2$ sweeps the frequencies from $1.5 \mathrm{~Hz}$ down to $0 \mathrm{~Hz}$ (Fig. 5-(a)).

- Two different random amplitude, random period (RARP) signals $R 1$ (for training) and $R 2$ (for validation), with maximum allowable switching period of $0.67 \mathrm{~s}$ and maximum allowable force amplitude of $1 \mathrm{kN} / \mathrm{m}$ (Fig. 5-(b)).

- Two different multisine signals $M 1$ (for training) and $M 2$ (for validation), consisting of 900 equally spaced frequencies in the range (1/600)-1.5Hz, with random phases and flat amplitude spectrum (Fig. 5-(c)).

All the experiments utilised in this paper have a time length of $600 \mathrm{~s}$ and a sampling period $T_{s}=0.1 \mathrm{~s}$. For further details regarding the utilised excitation signals, see [1].

\section{A. Model Identification}

Using the strategies illustrated in Section IV-C, the input delay time $n_{d}$ and the dynamical orders $n_{a}$ and $n_{b}$ are identified for both the $\eta \rightarrow y$ and $f_{I N} \rightarrow y$ model families.

In case of $\eta \rightarrow y$ class model, the loss function, calculated for the different ARX models, has no significant reduction for $n_{a}>8$, using a wide variety of $n_{b}$ and $n_{d}$ values, indicating that $n_{a}=8$ is the correct value to obtain a parsimonious model structure. Fig. 6 shows the loss function for different values of $n_{a}$, for the case of $n_{b}=2$ and $n_{d}=-7$. Similarly, given $n_{a}=8$, the loss function has no significant reduction for $n_{b}>2$, regardless of the value of $n_{d}$. Fig. 7 shows the loss function calculated for different $n_{d}$, for the case of $n_{a}=8$ and $n_{b}=2$. It is possible to see that the loss function minimum occurs for $n_{d}=-7$ (describing a noncausal ARX model).

Plots similar to Figs. 7 and 6 are obtained for the $f_{I N} \rightarrow y$ class model, with the results are summarized in Table I.

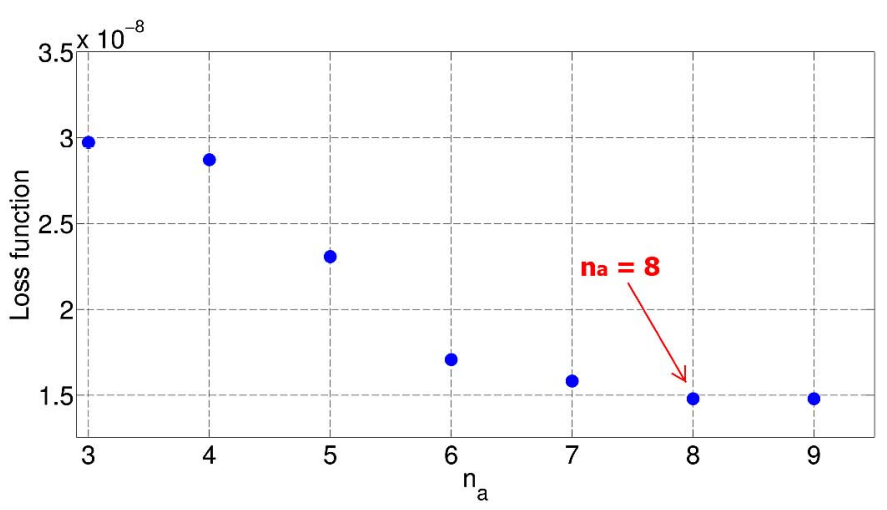

Fig. 6. Determination of the dynamical order $n_{a}=8$ in case of $n_{b}=2$ and $n_{d}=-7$, for the models belonging to $\eta \rightarrow y$. The NWT experiment $W 1$ has been used for the identification.

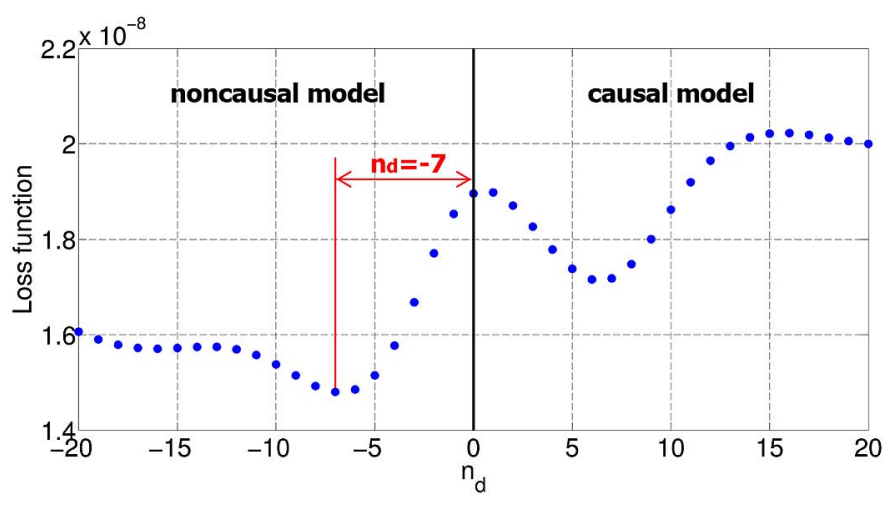

Fig. 7. Determination of $n_{d}=-7$ in case of $n_{a}=8$ and $n_{b}=2$, for the models belonging to $\eta \rightarrow y$. The NWT experiment $W 1$ has been used for the identification.

TABLE I

$n_{a}, n_{b}, n_{d}, p, n_{1}$ AND $n_{2}$ UtILISED FOR THE MODELS BELONGING TO $\eta \rightarrow y$ AND $f_{I N} \rightarrow y$

\begin{tabular}{|c|c|c|c|c|c|c|}
\hline MODEL & $n_{a}$ & $n_{b}$ & $n_{d}$ & $p$ & $n_{1}$ & $n_{2}$ \\
\hline$\eta \rightarrow y$ & 8 & 2 & -7 & 2 & 10 & 10 \\
\hline$f_{I N} \rightarrow y$ & 4 & 2 & 0 & 2 & 3 & 5 \\
\hline
\end{tabular}

The polynomial order of the KGP model $p=2$ is selected observing that bigger values of $p$ improve the training fitting but degrade the quality of the validation fitting (overfitting).

For the ANN structure, a good compromise between complexity and accuracy of the model has been found utilising $n_{1}=10$ and $n_{2}=10$ for $\eta \rightarrow y$ and $n_{1}=3$ and $n_{2}=5$ for $f_{I N} \rightarrow y$ (see Table I).

The model prediction $\hat{y}(k)$ is compared against the measured signal $y(k)$ using the normalised root mean-squared error (NRMSE) as a metric:

$$
N R M S E=\frac{\sqrt{\sum_{k}|y(k)-\hat{y}(k)|^{2}}}{\sqrt{\sum_{k}|y(k)|^{2}}}
$$

The NRMSE metric is selected over other metrics, such as the mean square error (MSE) or the mean absolute percentage error (MAPE), because the NRMSE is normalised with respect to the magnitude of $y(k)$ (unlike the MSE), and the NRMSE does not give a distorted picture of the error for $y(k)$ values close to zero (unlike the MAPE). 


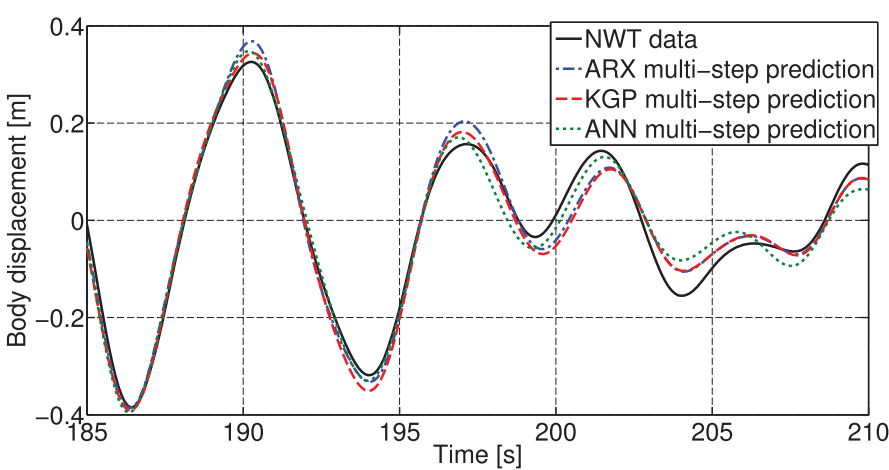

Fig. 8. Identified model performances ( $\eta \rightarrow y$ model family) on the training experiment $W 1$. The multi-step model predictions are compared with the training data.

TABLE II

NRMSE Multistep MOdel PERFormance For Training AND VALIDATION ( $\eta \rightarrow y$ MODEL FAMILY)

\begin{tabular}{|c|c|c|}
\cline { 2 - 3 } \multicolumn{1}{c|}{} & $\begin{array}{c}\text { Model trained } \\
\text { on } W 1\end{array}$ & $\begin{array}{c}\text { Model validated } \\
\text { on } W 2\end{array}$ \\
\hline ARX & 0.1721 & 0.1793 \\
KGP & 0.1713 & 0.1787 \\
ANN & 0.1671 & 0.2429 \\
\hline
\end{tabular}

TABLE III

NRMSE Multistep Model Performances for Training $\left(f_{I N} \rightarrow y\right.$ MODEL FAMILY $)$

\begin{tabular}{|c|c|c|c|}
\cline { 2 - 4 } \multicolumn{1}{c|}{} & \multicolumn{3}{c|}{ Model trained on } \\
\cline { 2 - 4 } \multicolumn{1}{c|}{} & $M 1$ & $R 1$ & $C 1$ \\
\hline ARX & 0.1018 & 0.0820 & 0.0799 \\
KGP & 0.0810 & 0.0684 & 0.0536 \\
ANN & 0.0408 & 0.0536 & 0.0442 \\
\hline
\end{tabular}

Fig. 8 shows, for the training experiment $W 1$, the multi-step predictions of the $\eta \rightarrow y$ class ARX, KGP and ANN models. All the fitting results on the training experiment $W 1$ are summarised in Table II. It is possible to observe only small differences in the performance of the linear ARX model compared to the two nonlinear models, indicating that the floating body motion does not exhibit much nonlinear behaviour for the geometry and wave conditions chosen in this case study. However, even for more energetic sea states (and, in particular, longer wave periods), within power production ranges, the device response to wave excitation alone does not tend to become significantly nonlinear, as documented in [23].

For the identification of the models belonging to $f_{I N} \rightarrow y$, the three different training experiments $M 1, R 1$ and $C 1$ are utilised. Table III reports the multi-step prediction performance of the models on the different training experiments, showing that the ANN models always performs the best for training data fitting, followed by the KGP model. The ARX model has consistently the worst training performance, suggesting that the data contain some nonlinearities, which the KGP model and the ANN model, in particular, are able to capture.

\section{B. Model Validation}

The $\eta \rightarrow y$ class models trained on the $W 1$ dataset, are validated on the $W 2$ dataset. Fig. 9 shows a comparison of the

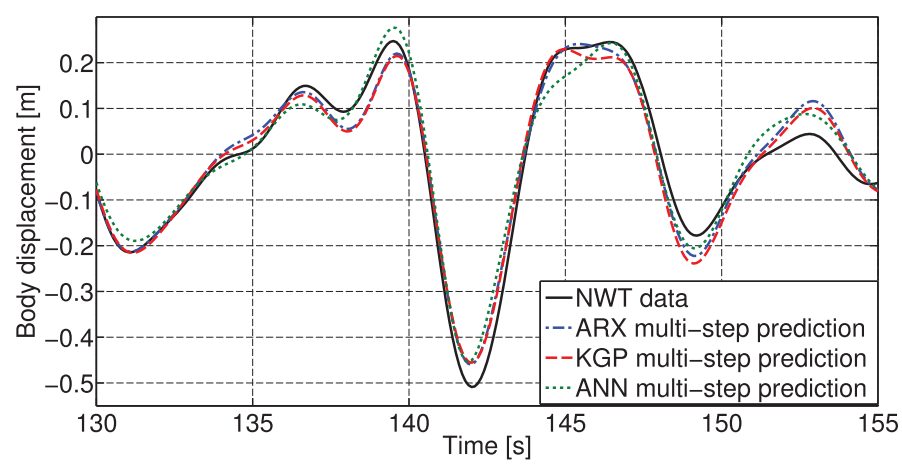

Fig. 9. Identified model performances ( $\eta \rightarrow y$ model family) on the validation experiment $W 2$. The models have been trained on the experiment $W 1$.

TABLE IV

NUMBER OF IDENTIFIED PARAMETERS FOR THE $\eta \rightarrow y$ AND $f_{I N} \rightarrow y$ MODEL FAMILIES

\begin{tabular}{|c|c|c|c|}
\cline { 2 - 4 } \multicolumn{1}{c|}{} & \multicolumn{3}{c|}{ Number of parameters } \\
\cline { 2 - 4 } \multicolumn{1}{c|}{} & ARX & KGP & ANN \\
\hline$\eta \rightarrow y$ & 11 & 22 & 241 \\
\hline$f_{I N} \rightarrow y$ & 7 & 14 & 50 \\
\hline
\end{tabular}

TABLE V

NRMSE MOdels Multistep PERformances FOR VALIDATION $\left(f_{I N} \rightarrow y\right.$ MOdEL FAMily $)$

\begin{tabular}{|c|c|c|c|c|c|}
\hline & \multicolumn{3}{|c|}{ Model trained on } \\
\hline & & & $M 1$ & $R 1$ & $C 1$ \\
\hline \multirow{9}{*}{ 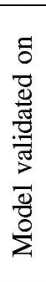 } & \multirow{3}{*}{ M2 } & ARX & 0.1005 & 0.0868 & 0.0988 \\
\hline & & KGP & 0.0807 & 0.0749 & 0.0748 \\
\hline & & ANN & 0.0414 & 0.0600 & 0.1162 \\
\hline & \multirow{3}{*}{$R 2$} & ARX & 0.1351 & 0.1240 & 0.1346 \\
\hline & & KGP & 0.1249 & 0.1192 & 0.1196 \\
\hline & & ANN & 0.1306 & 0.1099 & 0.1513 \\
\hline & \multirow{3}{*}{$C 2$} & ARX & 0.0828 & 0.0704 & 0.0757 \\
\hline & & KGP & 0.0654 & 0.0566 & 0.0513 \\
\hline & & ANN & 0.4572 & 0.1755 & 0.0497 \\
\hline
\end{tabular}

ARX, KGP and ANN model multi-step predictions against the validation data. The NRMSE performance for each of the different models against the validation data is summarised in Table II. The results show the ARX and KGP models maintain similar performance, if compared with their training performances, clear symptom that the models are able to generalise quite well and that the identification was correct. On the other hand, the ANN model, which was the best in training, degrades considerably in validation, showing an inability to generalise, likely a consequence of the nonlinear optimization necessary to identify the ANN parameters, which does not guarantee the achievement of a global minimum. Furthermore, as shown in Table IV, the number of parameters for the ANN model is considerably larger than the number of parameters for the ARX and KGP models, increasing the difficulty of finding a global minimum.

Table $\mathrm{V}$ shows the performance of the models belonging to $f_{I N} \rightarrow y$ on the validation experiments $M 2, R 2$ and $C 2$. Comparing the validation fitting of the ARX and KGP models with their training fitting, it is possible to see that the two models generalise quite well on $M 2$ and $C 2$, indicating good quality training data and model identification, but they show some more 
difficulty on $R 2$. On the other hand, the ANN model is less consistent exhibiting, in some cases, the ability to generalise well and in other cases an inability (training on $C 1$ and validation on $M 2$; training on $R 1$ and validation on $C 2$; all the validations on $R 2$ ). Once again, the results show the supplemental difficulty for the ANN identification, depending on the nonlinear optimization and larger number of parameters (Table IV). All the identified models exhibited more difficulties for validation on the experiment $R 2$, possibly due to the fact that RARP signals have very sharp corners, corresponding to fast changes in the applied PTO force and resulting body acceleration, which can create nonlinear effects.

\section{Conclusions}

A 2D circular device geometry has been modelled to describe its behaviour in power production mode, for a commonly occurring moderate sea state at the EMEC test site. Three discrete time model structures (ARX, KGP and ANN) have been introduced for the $\eta \rightarrow y$ and $f_{I N} \rightarrow y$ model families. In particular, for the $f_{I N} \rightarrow y$ case, the fitting performances of the nonlinear models always outperform the linear ARX model, showing the nonlinear nature of the NWT experiment data and the necessity of employing nonlinear models to describe the fluid and floating body interaction, when viscous effects are present and the body oscillations are not negligible compared to the body dimensions. This result has consequences for model-based control of WECs, whereby models determined from $\eta \rightarrow y$ cases, which may behave linearly, should not be used as a basis for model-based control design, due to the fact that the inclusion of the PTO control forces may drive the WEC into nonlinear operating regions.

The ANN model always displayed the best training performances, but, on the other hand, its ability in generalising on new experiments is not consistent, exhibiting the difficulties of nonlinear identification associated with neural networks.

The identified KGP nonlinear models exhibited good performances both in training and validation (especially for the $f_{I N} \rightarrow y$ model family), indicating that the training experiment data, the selected model structures and the identification procedures based on convex optimization are appropriate.

\section{REFERENCES}

[1] J. Davidson, S. Giorgi, and J. Ringwood, "Identification of wave energy device models from numerical wave tank data-Part 1: Numerical wave tank identification tests," IEEE Trans. Sustain. Energy, to be published.

[2] Z. Yu and J. Falnes, "State-space modelling of a vertical cylinder in heave," Appl. Ocean Res., vol. 17, no. 5, pp. 265-275, 1995.

[3] W. Cummins, "The impulse response function and ship motions," DTIC Document, Tech. Rep., 1962.

[4] R. Taghipour, T. Perez, and T. Moan, "Hybrid frequency-time domain models for dynamic response analysis of marine structures," Ocean Eng., vol. 35, no. 7, pp. 685-705, 2008.

[5] T. Pérez and T. I. Fossen, "Time-vs. frequency-domain identification of parametric radiation force models for marine structures at zero speed," Model. Ident. Control, vol. 29, no. 1, pp. 1-19, 2008.

[6] A. Bhinder, M. A. Babarit, L. Gentaz, and P. Ferrant, "Effect of viscous forces on the performance of a surging wave energy converter," in Proc. Int. Offshore Polar Eng. Conf. (ISOPE'12), 2012, pp. 545-549.

[7] A. Babarit and P. Laporte-Weywada, "On the numerical modelling of the nonlinear behaviour of a wave energy converter," in Proc. Int. Conf. Ocean Offshore Arctic Eng. (OMAE), 2009, CDROM.
[8] M. Guérinel, M. Alves, and A. Sarmento, "Nonlinear modelling of the dynamics of a free floating body," in Proc. Eur. Wave Tidal Energy Conf. (EWTEC), Southampton, U.K., 2011, CDROM.

[9] M. Lawson, Y.-H. Yu, A. Nelessen, K. Ruehl, and C. Michelen, "Implementing nonlinear buoyancy and excitation forces in the WECSim wave energy converter modeling tool," in Proc. 33rd Int. Conf. Ocean Offshore Arctic Eng., 2014, p. V09BT09A043.

[10] A. S. Zurkinden, F. Ferri, S. Beatty, J. P. Kofoed, and M. Kramer, "Nonlinear numerical modeling and experimental testing of a point absorber wave energy converter," Ocean Eng., vol. 78, pp. 11-21, 2014.

[11] L. Ljung, System Identification: Theory for the User, 2nd ed. Englewood Cliffs, NJ, USA: Prentice-Hall, 1999.

[12] L. Ljung, "Perspectives on system identification," Annu. Rev. Control, vol. 34, no. 1, pp. 1-12, 2010.

[13] T. Soderstrom and P. Stoica, System Identification. Englewood Cliffs, NJ, USA: Prentice-Hall, 1989.

[14] J. A. Armesto, R. Guanche, A. Iturrioz, C. Vidal, and I. J. Losada, "Identification of state-space coefficients for oscillating water columns using temporal series," Ocean Eng., vol. 79, pp. 43-49, 2014.

[15] J. Davidson, S. Giorgi, and J. V. Ringwood, "Linear parametric hydrodynamic models for ocean wave energy converters identified from numerical wave tank experiments," Ocean Eng., vol. 103, pp. 31-39, 2015.

[16] J. Falnes, "On non-causal impulse response functions related to propagating water waves," Appl. Ocean Res., vol. 17, no. 6, pp. 379-389, 1995.

[17] O. Nelles, Nonlinear System Identification: From Classical Approaches to Neural Networks and Fuzzy Models. New York, NY, USA: Springer, 2001.

[18] G. Golub and C. Van Loan, Matrix Computations, 4th ed. Baltimore, MD, USA: The Johns Hopkins Univ. Press, 2012.

[19] T. Sauer, Numerical Analysis. Newmarket, ON, Canada: Pearson Education Canada, 2012.

[20] L. Ljung, MATLAB System Identification Toolbox. Natick, MA, USA: MathWorks, 2013.

[21] M. F. Møller, "A scaled conjugate gradient algorithm for fast supervised learning," Neural Netw., vol. 6, no. 4, pp. 525-533, 1993.

[22] H. Demuth, M. Beale, and M. Works, MATLAB: Neural Network Toolbox: User's Guide. Natick, MA, USA: MathWorks, 1992.

[23] M. P. Retes, A. Mérigaud, J.-C. Gilloteaux, and J. V. Ringwood, "Nonlinear Froude-Krylov force modelling for two heaving wave energy point absorbers," in Proc. 9th Eur. Wave Tidal Energy Conf. (EWTEC), Nantes, France, 2015, pp 08C1-4-1-08C1-4-10.

Simone Giorgi received the Master's degree (first class Hons.) in electronics at the Universita degli Studi di Ferrara (UNIFE), Ferrara, Italy, in 2002, and the Master's degree (first class Hons.) in renewable energy systems from Maynooth University, Maynooth, Ireland. He is currently pursuing the Ph.D. degree at the Centre for Ocean Energy Research, Maynooth University. His research interests include nonlinear parametric modelling and control for wave energy devices using numerical tank testing.

Josh Davidson received the Bachelor's degree (first class Hons.) in physical sciences from James Cook University, Townsville City Qld., Australia, in 2006, and the Ph.D. degree in energy harvesting for marine-based sensors from James Cook University, Townsville City, Qld., Australia. He was cofunded by the Commonwealth Scientific and Industrial Research Organisation's (CSIRO) Energy Technology Division, working with their 'Energy Harvesting Team'. Currently, he works with the Centre for Ocean Energy Research, Maynooth University, Maynooth, Ireland, where his research explores nonlinear modelling for wave energy converters through numerical wave tank experiments.

John V. Ringwood (SM'97) received the Diploma degree in electrical engineering from Dublin Institute of Technology, Dublin, Ireland, and the Ph.D. degree in control systems from Strathclyde University, Glasgow, U.K., in 1981 and 1985, respectively. He is currently a Professor of Electronic Engineering with the National University of Ireland (NUI), Dublin, Ireland, and the Director of the Centre for Ocean Energy Research (COER), NUI Maynooth, Maynooth, Ireland. He was the Head of the Electronic Engineering Department, NUI Maynooth from 2000 to 2005, developing the Department from a greenfield site. His research interests include time series modelling, wave energy, and biomedical engineering. He is a Chartered Engineer. He is a Fellow of the Institution of Engineers of Ireland. 